\title{
REVISED
}

\section{USE OF THE IRDS STANDARD IN CALS}

\section{David K. Jefferson Cita M. Furlani}

U.S. DEPARTMENT OF COMMERCE Natlonal Instrtute of Standards and Technology

National Computer Systems Laboratory Gatthersburg, MD 20899 


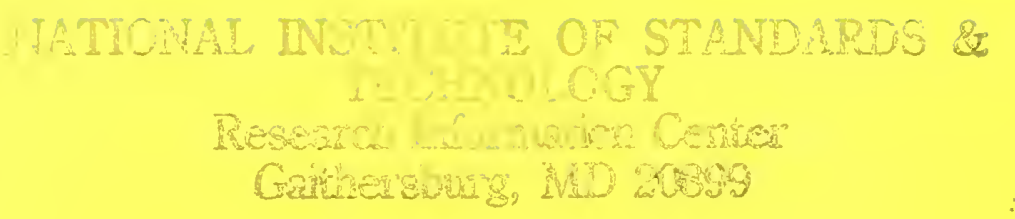




\section{USE OF THE IRDS STANDARD IN CALS}

\section{David K. Jefferson Cita M. Furlani}

U.S. DEPARTMENT OF COMMERCE Natlonal Ingtitute of Standards and Technology Natlonal Computer Systems Laboratory Galthersburg, MD 20899

September 1989

Revlsed November 1990

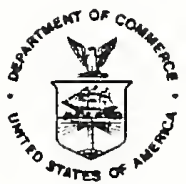

U.S. DEPARTMENT OF COMMERCE Robert A. Mosbacher, Secretary NATIONAL INSTITUTE OF STANDARDS AND TECHNOLOGY 
David K. Jefferson

Cita M. Furlani.

National Institute of Standards and Technology

\begin{abstract}
The objective of this point paper is to show how the Information Resource Dictionary system (IRDS) can fulfill critical design and operational requirements for CALS Phase II. First, a series of assumptions are made about the data management services which are needed by CALS Phase II. Next, these assumptions are used to develop a series of requirements for a dictionary system. The structure of the IRDS family of standards is then described. Examples are provided to illustrate how the IRDS could meet the requirements. A schedule is presented to show that the IRDS and other data management standards will be available when needed to meet the immediate requirements of CALS. An architecture is presented to illustrate additional standards required to achieve longerrange goals of distributed database. Finally, development tasks are recommended.
\end{abstract}




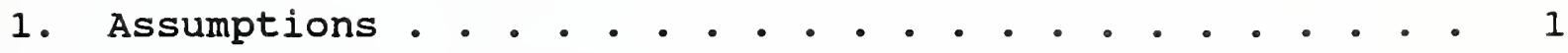

2. Requirements for a Dictionary • • • . . . . • • • • . 2

3. Structure of the IRDS Family of Standards . . . . . . . 3

3.1. ANS X3.138 . . . . . . . . . . . . . . . 3

3.2. IRDS Services Interface . . . . . . . . . . . . . 4

3.3. IRDS Export/Import File Format . . . . . . . . . 5

3.4. Categorization of the IRDS and other standards . 5

4. Availability of the IRDS and Data Management Standards 7

5. An Architecture for Distributed Database . . . . . 8

6. Development Tasks . . . . . . . . . . . . . . . 9

7. Bibliography • . . . . . . . . . . . . . . 10 
. 


\section{Assumptions}

The following assumptions are made about the mechanisms needed to achieve CALS Phase II:

- A logically integrated database is absolutely necessary.

- A single physical database is impractical--multiple physical databases controlled by multiple DBMSs are required.

- A three-schema architecture is the only practical way of achieving control over such a database.

- All activity of the DBMSs must be logically controlled by a logically single representation of the three-schema architecture.

- A single physical representation of the three-schema architecture is impractical--multiple, coordinated, geographically distributed physical representations are required.

- The development and maintenance of the three-schema architecture will be extremely complex and require a very large volume of data.

- To add further complication, a three-schema architecture, since it represents a union of different disciplines (as represented by different external schemas), may be developed using more than one modeling methodology. 


\section{Requirements for a Dictionary}

Given the preceding assumptions, the following may be concluded:

- A mechanism is required to actively supply three-schema information to the various DBMSs.

- A mechanism is required to coordinate separate sources of such three-schema information.

- A mechanism is required to support data modeling and schema maintenance.

- A mechanism may be required to support multiple methodologies for performing the data modeling process-i.e., there may be a requirement for a supporting mechanism that is neutral with respect to methodology.

Coordinated active dictionaries which are neutral with respect to data modeling methodology are one mechanism for satisfying the preceding requirements. Such dictionaries must support four distinct processes:

- They must have a standard interface to provide threeschema information to a variety of database management systems.

- They must support the process of coordinating communication to control the DBMSs.

- They must support the process of describing the vocabulary needed by the chosen data modeling methodologies.

- They must support the process by which each methodology populates a dictionary using its vocabulary. 


\section{3. structure of the IRDs Family of standards}

\subsection{AN8 83.138}

Most of the functionality of the IRDS is defined by the American National standard for Information Systems - Information Resource Dictionary system, which is an American National standard (ANS), X3.138-1988, and a Federal Information Processing standard (FIPS), Publication 156. The parts of this standard most relevant to CALS Phase II deal with the creation, maintenance, and retrieval of two distinct levels of data. One level of data is used to describe a vocabulary, and the other level is used to apply that vocabulary in the data modeling methodologies. The former level is referred to as the IRD Schema Level, and the latter as the IRD Level. The IRDS provides for extensibility at the IRD Schema Level to satisfy the particular requirements of any set of methodologies and applications. This degree of generality is not free: the first task in the use of the IRDS to support concurrent engineering must be to use the IRD Schema Level to define a standard vocabulary which will serve as a union among the individual methodologies and applications. Fortunately, the different data modeling methodologies have small vocabularies (e.g., a few different kinds of boxes, lines, and arrows) so this should not be a particularly difficult task. For example, the following vocabulary covers the major concepts of the IDEFIX modeling methodology:

- entity is an IDEFIX entity,

- entity-is-assembled-of-entity is a type of IDEFIX relationship,

- (cardinality -1 , cardinality-2) is a property of an IDEF1X relationship,

- entity-contains-relationship associates an attribute (or data element) with an entity; the association may have various properties (e.g., an attribute may be used-as "pk" to indicate that it is part of the primary key),

- category is an IDEFIX category,

- entity-is-a-category indicates that an entity is associated with a particular category, and

- category-set-is-total indicates that the set of entities is exhaustive.

The next task is then to use that vocabulary within the data modeling methodologies to define the three types of schemas. For example, the following objects serve to group entities into the indicated schemas: 
- conceptual-schema-view collects together the entities in the conceptual schema, and

- LSA-external-schema-view collects together the entities in the external schema used for LSA.

The following are examples of entities, relationships, and attributes in a schema:

- product-item is-assembled-of product-item-usage cardinality $=$ (parent $=1 "$, child="n") indicates that one item may be assembled from many simpler items,

- product-item contains part-number used-as = "pk" indicates that the primary key of item is the part number, and

- geometric-model is-a geometric-model-category geometricmodel-category can-be-a wire-frame-model indicates that a geometric model can be a wire frame model or some other type of model which may or may not be enumerated explicitly.

A particularly important feature of the IRDS is the ability to provide user-oriented names. For example, associated with product-item may be the following:

- identification-names $=$ (alternate-name = "LSA product item", alternate-name-context = "LSA"), which could provide a special name for product-item in the LSA external schema,

- descriptive-name = some-very-long-descriptive-namewhich-is-readable-but-not-easily-typed-or-accepted-byprogramming-languages-or-DBMSs, provides an alternate to the normal short access name, and

- some-general-name (LSA-SOL-variation: 2) indicates that this is revision 2 of a general object which has been specialized to the use of SQL in an ISA application.

\subsection{IRDs services Interface}

ANS $\times 3.138$ defines two interfaces which can be used by people to define and populate a repository for schema definitions, but there are no standard capabilities for constructing analyses, diagrams, and so on for the schemas. This is appropriate, given that there are no standard methodologies. other tools, dependent on particular methodologies, must request data from the IRDS to provide these capabilities. The draft standard for the IRDS Services Interface defines functionality for communicating between the IRDS and other software, such as a diagramming 
program or a DBMS. The Services Interface is a program call interface which complements the human interfaces defined in ANS $\mathrm{X} 3.138$. This interface will provide the active dictionary support that is required to control the DBMS, as well as allowing continued use and integration of existing support tools for IDEF0, IDEF1X and other methodologies.

\subsection{IRDS Export/Import File Format}

ANS $\mathrm{X} 3.138$ also specifies functionality for exporting all or part of a dictionary into a file, checking a second dictionary for compatibility with that file, and optionally importing that file into the second dictionary. However, ANS X3.138 does not specify the format of the file; this is the subject of the final critical element in the IRDS family of standards, the draft standard for the IRDS Export/Import File Format. This defines an efficient and reliable format for communicating mass data, such as an entire database schema or collection of database schemas, from one dictionary to another. ANS X3.138 in conjunction with the IRDS Export/Import File Format standard can be used to compare one dictionary with another, as well as to populate an empty dictionary. The standards will therefore provide the required capability of dictionary coordination.

\subsection{Categorization of the IRDs and other standards}

The standards needed by CALS Phase II can be divided into two general categories:

- Data standards represent the "business rules" of CALS, and are developed by the CALS office, the military Services and Agencies, and defense contractors. They are represented by the contents of two layers of the IRDS :

○o IRD Schema Layer represents modeling rules, and

oo IRD Layer represents the schemas and related information.

- Technical standards represent the standard "tools" for building CALS database systems. These are currently the following:

○० IRDS ANS $\times 3.138$,

oo proposed IRDS Services Interface,

oo proposed IRDS Export/Import File Format, 
oo SQL for relational database management at a particular site, and

oo proposed Remote Database Access (RDA) for executing a query at a remote site. 


\section{Availability of the IRDs and Data Management standards}

The diagram on Timelines of Data standards Needed by CALs and PDES is intended to demonstrate that data management standards and commercial implementations are expected to be available when they are needed to satisfy CALS requirements. SQL is evolving through SQL2 to SQL3, which is expected to be object-oriented and capable of effectively managing text, graphics, and other CALS data types. Remote Database Access (RDA) will provide a primitive capability for sending a query to a remote database and receiving data and status information in response.

\begin{tabular}{|c|c|c|c|c|c|c|}
\hline & 1989 & 1990 & 1991 & 1992 & 1994 & 1995 \\
\hline LSAR & $\begin{array}{l}\text { SQL } \\
\text { Schema }\end{array}$ & & & \multirow{2}{*}{\multicolumn{3}{|c|}{$\begin{array}{l}\text { LSAR/PDES } \\
\text { Integration }\end{array}$}} \\
\hline PDES & \multicolumn{2}{|c|}{$\begin{array}{l}\text { PDES } \\
\text { Integration }\end{array}$} & $\begin{array}{l}\text { PDES } \\
1.0 \\
\text { std. }\end{array}$ & & & \\
\hline 8QL & \multicolumn{3}{|c|}{$\begin{array}{l}\text { SQL } \\
\text { Software }\end{array}$} & \multicolumn{2}{|l|}{$\begin{array}{l}\text { SQL2 Std. and } \\
\text { Software }\end{array}$} & $\begin{array}{l}\text { SQL3 } \\
\text { std. }\end{array}$ \\
\hline RDA & & & & \multicolumn{3}{|c|}{ RDA std. and Software } \\
\hline IRDS & std. & $\begin{array}{l}\text { Soft- } \\
\text { ware }\end{array}$ & $E / I$ & \multicolumn{3}{|c|}{$\begin{array}{l}\text { Services Interface } \\
\text { Standards and Software }\end{array}$} \\
\hline
\end{tabular}

Timelines of Data standards Needed by CALS and PDES 


\section{An Architecture for Distributed Database}

RDA is insufficient for true distributed database management, since it does not deal with such issues as query decomposition (where a single query requests data from multiple databases) or transaction management (needed to resolve simultaneous accesses and updates to the same data). The following is a possible layered architecture for standards that would provide the required services:

\section{DBMS syntax and presentation transparency \\ 5. location and performance transparency \\ 4. transaction transparency \\ 3. communication transparency \\ 2. mapping from global conceptual schema into local internal schema \\ 1. database access}

The users interact with Layer 6, which isolates them from details of names, formats, and DBMS syntax at the various databases. Each user seems to be dealing with a single, dedicated database specific to his or her requirements. IRDS support of a threeschema architecture is essential to map from the user's local external schema into the global conceptual schema. Layer 5 performs services such as query decomposition and optimization, in order to effectively deal with data distribution. Directory and schema information is essential. Layer 4 provides for detection and resolution of conflicts in accessing and updating data. Layer 3 handles the details of communicating with remote sites. RDA is positioned in Layer 3. Layers 2 and 1 provide services at a particular remote site to satisfy a particular piece of the original query or update. IRDS services are again required to provide support for the three-schema architecture. New standards will be required for Layers 6, 5, 4, and 3, while IRDS and SQL may require extensions to deal with Layer 2 . Layer 1 provides operating system services that are identical for distributed or centralized databases. 


\section{Development Tasks}

The following are some of the near-term tasks required for CALS Phase II:

- Development of the vocabulary at the IRD Schema Layer to support methodologies for modeling the various applications.

- Development of the vocabulary at the IRD Schema Layer to support modeling distributed databases.

- Population of the IRD Layer with application models.

- Population of the IRD Layer with distributed database models.

- Interfacing Computer-Aided Software Engineering (CASE) tools to the IRDS, in order to assist in development and analysis of models.

- Development of standard ways of using the IRDS to support integrated process and data modeling (e.g., through a standard based on IDEFO and IDEF1X).

The following are some of the longer-term tasks required for CALS Phase II:

- Completion of the architecture for distributed database.

- Development of standards based on that architecture.

The conclusion is that the IRDS is a good basis for CALS Phase II, but a large amount of work is needed to develop other standards that will be required. 


\section{Bibliography}

Goldfine, Alan and Konig, Patricia. A Technical Overview of the Information Resource Dictionary System (Second Edition), NBSIR 88-3700, January 1988, $142 \mathrm{p}$.

Goldfine, Alan. Using the Information Resource Dictionary System Command Lanquage (Second Edition), NBSIR 88-3701, January 1988, $83 \mathrm{p}$.

Goldfine, Alan and Kirkendall, Thomasin. The ICST-NBS Information Resource Dictionary System Command Lanquage Prototype, NBSIR 88-3830, August 1988, 55 p.

Law, Margaret Henderson. Guide to Information Resource Dictionary System Applications: General Concepts and Strategic systems Planning, NBS SP 500-152, April 1988, 145 p. 


\section{BIBLIOGRAPHIC DATA SHEET}

2. PERFORMIMO ORQAMIZATION REPOAT NUMBER

3. PUBLCATION DATE

September 1989

4. TITLE AND SUBTITLE

Use of the IRDS Standard in CALS

5. AUTHOR(S)

David $K$. Jefferson and Cita M. Furlani

6. PEAFOAMINO ORQANIZATION (IF JOINT OA OTHEA THAN NIST, SEE INSTAUCTIONS) U.S. DEPARTMENT OF COMMERCE

MATIONAL INSTITUTE OF STANDARDS AND TECHNOLOGY

GATHEASEUAQ, MO 20899

7. CONTRACT/GRANT NUMBEA

8. TYPE OF REPORT AMD PEAIOO COVERED

9. SPONSORIMG ORGAMIZATION NAME AND COMPLETE ADDRESS (STREET, CITY, STATE, ZIP)

10. SUPPLEMENTAAY NOTES

DOCUMENT DESCRIBES A COMPUTER PROGRAM: SF.185, FIPS SOFTWARE SUMMARY, IS ATTACHED.

11. ABSTRACT (A 200-WOAD OA LESS FACTUAL SUMMARY OF MOST SIONIFICAMT INFORMATION. IF DOCUMENT INCLUDES A SIONIFICANT BIBUOGRAPHY OR UTERATURE SUAVEY, MENTION IT HERE.)

The objective of this point paper is to show how the Information Resource Dictionary System (IRDS) can fulfill critical design and operational requirements for CALS Phase II. First, a series of assumptions are made about the data management services which are needed by CALS Phase II. Next, these assumptions are used to develop a series of requirements for a dictionary system. The structure of the IRDS family of standards is then described. Examples are provided to illustrate how the IRDS could meet the requirements. A schedule is presented to show that the IRDS and other data management standards will be available when needed to meet the immediate requirements of CALS. An architecture is presented to illustrate additional standards required to achieve longer-range goals of distributed database. Finally, development tasks are recommended.

12. KEY WOADS (8 TO 12 ENTRIES; ALPHABETICAL ORDER; CAPITALLZ ONLY PNOPER MAMES; AND SEPARATE KEY WORDS BY SEMICOLONS) Computer-aided Acquisition and Logistic Support (CALS); data modeling; distributed data management; Information Resource Dictionary System (IRDS); Remote Database Access (RDA); SQL

13. AVAILABIUTY

$\mathrm{X}$ UNLMATED

FOR OFFICIAL DISTAIBUTION. DO NOT RELEASE TO NATIONAL TECHMICAL INFORMATION SERVICE (NTIS).

ORDER FROM SUPERINTENDENT OF DOCUMENTS, U.S. GOVERNMENT PAINTINO OFFICE, WASHINOTON, DC 20402.

ORDER FROM NATIONAL TECHNICAL INFORMATION SERVICE (NTIS), SPRINGFIELD, VA 22161.

14. NUMBER OF PRINTED PAGES

15. PRICE

$\mathrm{AO} 2$ 

' 
